\title{
A review on the functional properties of fermented soymilk
}

\author{
Periyanaina KESIKA ${ }^{1}$, Bhagavathi Sundaram SIVAMARUTHI ${ }^{1}$, Chaiyavat CHAIYASUT $^{1 *}$
}

\begin{abstract}
Fermented soybean products have been used for several centuries. Fermented soymilk is one of the known soy products with several health benefits. The current literature review recapitulates the various health benefits of fermented soymilk. In vitro and in vivo studies have shown that the consumption of fermented soymilk protects one from cancer, diabetes, hyperlipidemia, etc. It has also been reported that fermented soymilk has immune-modulatory, memory improvement, anti-colitis, and wound healing activities. However, there are few studies to confirm the health benefits of fermented soymilk for human subjects. The dose and duration of the intervention may vary between the animal model and humans. So, additional studies are obligatory to authorize the proposed health benefits of fermented soymilk.
\end{abstract}

Keywords: soymilk; fermented soymilk; lactic acid bacteria; Lactobacillus; Bifidobacterium.

Practical Application: The review may help researchers to develop soybean-based functional foods that are effective against several illnesses.

\section{Introduction}

Soy products have been used for ages in various forms, including soymilk, soy cheese, tempeh, tamari, soy sauce, soy yogurt, and soy mayonnaise. They are rich in isoflavones, and their consumption is associated with several health benefits, especially protection from gastrointestinal disorders, cardiovascular diseases, and cancers (Asif \& Acharya, 2013; Zhang et al., 2003; Messina, 2016; Pisani et al., 1999).

Soy products are commonly produced by fermentation process, which improves the bioavailability of bioactive compounds and enhances the phytochemical property and sensory quality of soy products. Lactic acid bacteria (LAB) are generally used for the production of fermented soy products. Specifically, Bacillus subtilis, Lactobacillus spp., and Bifidobacterium spp. are commonly used to produce fermented soy products (Jayachandran \& Xu, 2019).

Fermented soymilk is one of the soybean-based functional foods with known health benefits. The microbes used for the production of fermented soymilk play a critical role in its end products. The microorganisms that produce $\alpha$-galactosidase and $\beta$-glucosidase can hydrolyze raffinose and stachyose and catalyze the hydrolysis of $\beta$-glucosides. Isoflavones are the major bioactive chemicals in soybean, which are available in four various forms: aglycones, $\beta$-glucosides, $6 "$-O-malonylglucosides, and 6"-O-acetylglucosides (Wang, 2008).

The aglycone form of isoflavones can easily be absorbed by the human body and confers more health benefits than other glycosylated forms (Baú et al., 2015). Fermented soymilk can be produced with a high content of isoflavone aglycones by using a microorganism that can produce $\beta$-glucosidase (Yeom et al., 2012).
Though several studies are available on the production and functional properties of soy products, there is no concise literature review on the functional properties of fermented soymilk. Thus, the current manuscript summarizes the reported functional properties of fermented soymilk. The scientific literature was collected from Scopus, PubMed, Google Scholar, and ResearchGate using the keyword "Fermented soymilk." The results were manually screened for the suitability of the report. The appropriate research outputs were selected for the preparation of the manuscript.

\section{Health benefits of fermented soymilk}

\subsection{Antioxidant activity}

The soymilk fermented with Lactobacillus acidophilus CCRC 14079 or Streptococcus thermophiles CCRC 14085 and Bifidobacterium infantis CCRC 14633 or B. longum B6 exhibits radical scavenging activity against superoxide and peroxide and also inhibits the autoxidation of ascorbate. The bioactivity of the soymilk was high when it was fermented with both LAB and Bifidobacterium compared to when it was fermented with a single strain. Also, fermented soymilk has higher antioxidant capacity than unfermented soymilk (Wang et al., 2006).

The in vitro study revealed that Bacillus subtilis 2829PNU015 mediated fermented soymilk has high antioxidant capacity in 1,1-Diphenyl-2-picrylhydrazyl (DPPH) scavenging and low-density lipoprotein (LDL) oxidation inhibition assays compared to that of the unfermented soymilk (Seo et al., 2009).

L. rhamnosus CRL981 mediated fermentation process increased the antioxidant capacity of soymilk. The assessment of 
the antioxidant capacity of the fermented soymilk via $\beta$-carotene bleaching and DPPH methods showed that CRL981 mediated fermentation process improved the bioactivity of the soymilk. Moreover, the capability of CRL981 to produce $\beta$-glucosidase increased the isoflavone aglycones content in the fermented soymilk (Marazza et al., 2012).

Soymilk fermented using Streptococcus thermophilus grx02 showed higher antioxidant activity in DPPH assay and inhibited the formation of malondialdehyde (MDA). The supplementation of fermented soymilk significantly reduced alanine transaminase (ALT), aspartate transaminase (AST) activities in alcohol-fed mice. The content of glutathione and alcohol dehydrogenase, superoxide dismutase activities were increased in the liver of the mice supplemented with fermented soymilk compared to the control (Xu et al., 2012).

Soymilk fermented with $L$. paracasei KUMBB005 showed high antioxidant activity in DPPH and ferric reducing antioxidant power (FRAP) assays (Usha Rani \& Pradeep, 2015). Similarly, soymilk fermented with $L$. fermentum LAB 9 displayed more significant antioxidant activity in DPPH assay compared to control (Fakri et al., 2016).

Yamamoto et al. reported that soymilk fermented with Leuconostoc mesenteroides MYU 60 or Pediococcus pentosaceus MYU 759 or Lactobacillus plantarum MYU 74 or Lactobacillus reuteri MYU 220 improved greatly in reducing the production of reactive oxygen species. Notably, soy milk fermented with P. pentosaceus MYU 759 had more antioxidant capacity than the other fermented soymilks and control (Yamamoto et al., 2019).

L. casei 16 mediated fermentation process improved the content of total phenolic, isoflavone aglycones, and free amino acids in soymilk. The fermented soymilk extract protected the $\mathrm{HepG}_{2}$ cells against 2,2'-azobis (2-amidinopropane) dihydrochloride-induced damages (Qian et al., 2020).

\subsection{Anti-proliferative activity}

The anti-mutagenic activity of soymilk fermented with S. thermophilus and B. infantis (Hsieh \& Chou, 2006; Hsieh et al., 2007), L. acidophilus, and B. longum (Hsieh \& Chou, 2006) has been studied. The anti-mutagenic property of the soymilk increased after its fermentation. The degree of its bioactivity depended on the starter culture used for the fermentation process and the type of mutagen tested. The fermented soymilk showed more significant anti-mutagenic activity against 3,2' - dimethyl-4amino-biphenyl, a known potent mutagen. S. thermophilus and $B$. infantis mediated fermentation process effectively improved the anti-mutagenic property of soymilk against 4-nitroquinoline- $\mathrm{N}$ oxide and 3,2'- dimethyl-4-amino-biphenyl mediated mutagenicity (Hsieh \& Chou, 2006; Hsieh et al., 2007).

The fermented soymilk was prepared using Weissella cibaria FB069 or L. rhamnosus GG as a starter culture. A synbiotic formulation was made with FB069 or GG mediated fermented soymilk and xylooligosaccharides (XOS). The addition of XOS improved the dextran, folate, $\gamma$-aminobutyric acid, and aglycone content in soymilk fermented with FB069. The synbiotic fermented soymilk effectively suppressed the proliferation of HCT116 and Caco- 2 cell lines. The anti-proliferative property of the synbiotic soymilk was associated with TLR4/NF- $\kappa B$ signaling pathway. The expression of MyD88, TLR4, NF- $\mathrm{kB}$, and MD2 was reduced in synbiotic soymilk treated in HCT116 cells and Caco-2 (Le et al., 2020). Similarly, Qian et al. reported the anti-proliferative activity of ethanolic extract and water extract of soymilk fermented with $L$. casei 16 in HT-29 cells and Caco- 2 cells. The inhibitory activity was in a dose-depended manner. Both extract treatment suppressed the proliferation of cell nuclear antigen (it plays a role in cell cycle regulation and DNA replication) expression in HT-29 cells (Qian et al., 2020).

Japan Public Health Center-based Prospective study did not report that there is any link between consuming soy products (both fermented and unfermented soymilk) and liver cancer risk (Abe et al., 2020).

\subsection{Anti-diabetic activity}

Kwon et al. reported that fermented soymilk prepared with Kefir culture and Rhodiola extracts could improve type II diabetic condition. The in vitro study showed that the fermented soy milk reduced $\alpha$-amylase inhibitory activity. The fermentation process enriched the product with bioactive phytochemicals like polyphenolic compounds (Kwon et al., 2006).

Soymilk fermented with Bacillus subtilis improved the diabetic condition in streptozotocin-induced diabetic mice via $\alpha$-glucosidase and $\alpha$-amylase inhibition. The protective activity was higher in fermented soymilk compared to unfermented soymilk (Yi et al., 2009). Similarly, Ju and Han demonstrated that the soymilk fermented together with bokbunja (Rubus coreanus Miquel) extract using B. subtilis exhibited more inhibitory activity against $\alpha$-glucosidase than the soymilk fermented alone. $5 \%$ treatment of fermented soymilk with bokbunja extract significantly reduced the high postprandial blood glucose levels in diabetic mice model. The results suggested that the addition of other phytochemical-rich fruit preparation could increase the protective nature of the fermented soymilk (Ju \& Han, 2010).

L. rhamnosus CRL981 mediated fermented soymilk significantly alleviates the consequences of diabetes and improved the lipid profile and total antioxidant capacity in streptozotocin-induced diabetic mice. The bioactivity was possibly attributed to the high isoflavones content and high $\beta$-glucosidase activity. The study claimed that the selection of appropriate starter culture for the production of fermented soy products is an important key factor for developing functional food products (Marazza et al., 2013).

The supplementation of soymilk (SM), fermented soymilk (FSM), and fermented soymilk with omega-3 (FSMO3) significantly reduced the blood glucose level, total cholesterol, triglyceride, and malondialdehyde in streptozotocin-induced diabetic mice. Notably, FSMO3 intervention improved the body weight, SOD activity, hemoglobin concentration, and red blood cells count in diabetic mice. The study results suggested that FSMO3 have protective action against diabetic condition (Mohammadi Sartang et al., 2015). 
Soymilk fermented together with Cuminum Cyminum essential oil (FSMC) using L. plantarum A7 improved the fasting blood glucose level and total cholesterol content in diabetic rat model. Also, the levels of low-density lipoprotein cholesterol and high-density lipoprotein cholesterol were decreased and increased, respectively, in diabetic rats supplemented with FSMC (Babashahi et al., 2020). The supplementation of soy yogurt reduced the blood glucose level and improved the expression of Glut4 in rats. The study supports that the regular consumption of fermented soy products could provide health benefit (Setiawan et al., 2020).

\subsection{Immunomodulatory Properties}

Soymilk fermented with L. helveticus R0052, B. longum R0175, and S. thermophilus ST5 have been reported to have immune enhancing property. The soy milk treated with mixed and single strains reduced the production of IL-8 in tumor necrosis factor- $\alpha$ (TNFa)-treated intestinal epithelial cells (Wagar et al., 2009).

Sirilun et al. reported that the soymilk fermented using lactic acid bacteria increased the expression of IL-10 in vitro. The study claimed that probiotic-lactic acid bacteria mediated fermented soymilk could be used as food supplement to improve immune health (Sirilun et al., 2017).

\subsection{Anti-inflammatory Activity}

The methanol extract of Streptococcus salivarius subsp. thermophilus CCRC 14805 and/or B. infantis CCRC 14603 mediated fermented soymilk showed anti-inflammatory activity in vitro. The fermented soymilk extract inhibited iNOS expression in lipopolysaccharide (LPS)-induced macrophages. The fermented soymilk extract suppressed the expression of TNF- $\alpha$, IL-6, IL-11 $\beta$, and PGE2 (prostaglandin E2) in LPS-induced RAW 264.7 macrophages (Liao et al., 2010). Wu et al. also reported that L. plantarum mediated fermented soymilk has anti-inflammatory activity in LPS-induced macrophages. The protective action was possible through inhibition of NF- $\kappa \mathrm{B}$ activation. In detail, fermented soymilk treatment reduced the expression of TNF- $\alpha$, IL-6, and IL- $1 \beta$. The fermented soymilk attenuated the production of NO, PGE2, and reactive oxygen species (ROS) in LPS-induced macrophages (Wu et al., 2013).

\subsection{Antilipidemic activity}

Leuconostoc kimchii, Leuconostoc citreum, and L. plantarum mediated fermented soymilk (Soypro ${ }^{\mathrm{TM}}$ ) intervention reduced the total cholesterol (TC), LDL-cholesterol (LDL-C) levels in high-fat diet-induced obese rats. The triglyceride (TG) and high-density lipoprotein cholesterol (HDL-C) values were not changed in Soypro ${ }^{\mathrm{TM}}$ supplemented group compared to the control. The in vitro experiments showed that the cellular TG level was reduced in adipocyte 3T3-L1 cells upon Soypro ${ }^{\mathrm{TM}}$ treatment (Kim et al., 2008).

Soymilk fermented with or without Momordica charantia using L. plantarum NTU 102 or L. paracasei subsp. paracasei NTU 101 was prepared. The preparation contained $25 \%$ of milk to increase the acceptability of the fermented product. The anti-atherosclerosis property of the preparation was tested using hyperlipidemic hamsters. The test animals were supplemented with fermented soymilk product for eight weeks. The results showed that the NTU 102 mediated fermented soymilk supplementation significantly reduced the ratio of LDL-C/HDL-C; whereas, NTU 101 mediated fermented soymilk with $M$. charantia increased the SOD and TAC of the animals. The study concluded that NTU 101 mediated fermented soymilk with or without $M$. charantia can prevent hyperlipidemia-induced atherosclerosis and oxidative stress (Tsai et al., 2009).

The consumption of soy product (soy yogurt) fermented by Lactobacillus delbrueckii subsp. delbrueckii SNC33 reduced the liver weight and hepatic TG levels in the rat model. Further, the soy yogurt intervention reduced the plasma cholesterol level and increased the expression of $\beta$-oxidation-related genes in rat liver. Notable down-regulation of the SREBP-1 gene and lipogenesis-associated enzymes was observed in the group treated with soy yogurt compared to the control. The results suggested that soy yogurt consumption could prevent hepatic lipid accumulation (Kitawaki et al., 2009). The SNC33 mediated fermented soy product intervention upregulated the CYP7al expression and suppressed the fatty acid synthesis related genes and SREBP-2 in rats. The protective action was noticed after five weeks of supplementation and was observed only in the fermented soymilk-treated group (Kobayashi et al., 2012a; Kobayashi et al., 2012b).

An eight-week supplementation of Bacillus subtilis KCCM42923 mediated fermented soymilk reduced the plasma TC, LDL-C levels in apolipoprotein E knockout mice. In contrast, body weight, organ weight, TG, and HDL-C levels of the mice were not affected. The results showed that the KCCM42923 mediated fermented soymilk helps to control the plasma cholesterol level (Seo et al., 2010).

L. plantarum P-8 mediated fermented soymilk (LP8FS) intervention significantly increased the HDL-C level and reduced the TC and LDL-C levels in hyperlipidemic experimental rats. The fecal excretion of bile acids, TG, and TC was also observed in the LP8FS treatment group. The LP8FS treated group had improved liver function and reduced oxidative stress. The LP8FS intervention improved lipid metabolism and positively altered the gut microbiota in hyperlipidemic rats (Wang et al., 2013). Likely, L. plantarum A7 mediated fermented soymilk (LA7FS) intervention also improved the HDL-C level in streptozotocinnicotinamide-induced diabetic Wistar rats. In comparison, there were no significant changes in LDL-C and other biomarkers among the LA7FS group and control (Babashahi et al., 2015).

Lactococcus acidophilus MF204 mediated fermented soymilk (MFFSM) treatment regulated the lipid levels and normalized the TG, HDL-C, and LDL-C levels in hyperlipidemic rats. Arteriosclerosis and coronary risk indexes were reduced in MFFSM treated group compared to the other groups. The total antioxidant capacity of rats given MFFSM supplement was improved (Chen et al., 2017). 
Probiotics (L. plantarum, L. casei, and B. bifidum) mediated fermented soymilk (PFSM) was prepared and its hypolipidemic property was evaluated. PFSM intervention significantly reduced the high-fat diet-induced weight gain, liver fat accumulation, ROS production, and hyperlipidemia in the mice model. Additionally, the intervention of PFSM reduced the ALT, LPS, and TNF- $\alpha$ levels and increased the serum leptin level. The results showed that the supplementation of PFSM could protect the high-fat diet-induced consequences in mice (Zhang et al., 2017).

The fermented soymilk treatment significantly reduced the lipid accumulation in palmitic acid-induced lipogenic HepG2 cells. The suppression of SREBP-1 expression, induction of NRF-2 expression, and increased phosphorylation of ERK were observed in fermented soymilk treated cells. The fermented soymilk reduced the palmitic acid-induced ROS generation in HepG 2 cells. The results indicated that FSM could prevent lipid accumulation and reduce ROS generation (Ahn et al., 2018).

L. acidophilus and L. casei mediated fermented soymilk supplementation significantly reduced the plasma glucose level and improved the insulin level in hypercholesterolemic rats. Fermented soymilk intervention increased the HDL level and decreased the LDL level. The safety study suggested that fermented soymilk consumption was safe and helped to prevent cardiovascular diseases and manage metabolic disorders (Ahsan et al., 2019).

\subsection{Skin health}

The hairless ovariectomized mice were fed with fermented soymilk. The fermented soymilk supplementation effectively reduced the epidermal thickness and erythema intensity of dorsal skin and also reduced the cyclobutane pyrimidine dimers levels in dorsal skin. The isoflavone levels were increased in dorsal skin and serum in fermented soymilk supplemented mice compared to control. The study claimed that the supplementation of fermented soymilk protects the skin from UV irradiation and photo-damage in experimental hairless mice (Kano et al., 2016).

B. breve Yakult and L. mali mediated fermented soymilk consumption improve facial wrinkles in healthy women. An eight-week intervention study showed that fermented soymilk consumption significantly improved the average depth and maximum depth of skin wrinkles in study subjects. The urine isoflavone level was also increased in the treatment group compared with the control, which indicates that the fermented soymilk consumption aid in delaying skin aging (Kano et al., 2018).

The supplementation of fermented soymilk containing L. casei Shirota improved the skin conditions in terms of elasticity, dryness, pigmentation, coarseness, moisture, and stratum corneum morphology. The urine isoflavone level was increased, and the abundance of Lactobacillaceae and Bifidobacteriaceae was also increased in the treatment group. The level of Enterobacteriaceae and Porphyromonadaceae were reduced significantly after the consumption of fermented soymilk. The study suggested that fermented soymilk's regular consumption confers skin health to premenopausal healthy women (Nagino et al., 2018).

\subsection{Other bioactivities}

The whey obtained from LAB (L. casei, L. acidophilus, L. bulgaricus, S. thermophilus, and B. longum) mediated fermented soymilk (rich in peptides and $\gamma$-aminobutyric acid) was administrated to the spontaneously hypertensive rat for eight weeks. The intervention significantly reduced the systolic blood pressure in the experimental rats (Tsai et al., 2006).

L. plantarum CCFM8610 or L. bulgaricus CCFM8004 mediated fermented soymilk was given as supplement to the experimental mice with or without cadmium for eight weeks. CCFM8610 fermented soymilk facilitates the fecal cadmium excretion, increased the antioxidant capacity, and reversed hepatic and renal damage effectively than non-fermented soymilk and CCFM8004- fermented soymilk. The results suggested that CCFM8610-fermented soymilk could protect from chronic cadmium toxicity (Zhai et al., 2015).

L. fermentum LAB 9- fermented soymilk significantly improved the LPS-induced memory defects in experimental mice (Fakri et al., 2016). L. plantarum TWK10- fermented soymilk could enhance learning and memory in deoxycorticosterone acetate-salt hypertension-induced vascular dementia rats (Liu et al., 2016). Miyake et al. reported that the consumption of fermented soy products effectively lowers depressive symptoms during pregnancy (Miyake et al., 2018).

The ethanol extract of TWK10- fermented soymilk (ETFSM) effectively reduced oxidative stress in vitro. ETFSM intervention significantly exhibited the wound healing activity in streptozotocin-induced diabetic rats and decreased inflammation-associated proteins. The results indicated that the topical application of ETFSM helps to accelerate wound healing (Chuang et al., 2019). The administration of ETFSM and water extract of TWK10- fermented soymilk enhances the antioxidant capacity and improves cognitive function in type 2 diabetic rats (Liu et al., 2020).

L. fermentum BM-325 used for fermenting soymilk increased its bioactivity. $\beta$-glucosidase, $\alpha$-galactosidase, antioxidant activity, and angiotensin-converting enzyme inhibitory activities improved in soymilk after its fermentation. The results suggested that BM-325 mediated fermentation could increase the bioactivity of soymilk (Myagmardorj et al., 2018).

The protective nature of riboflavin-producing $L$. plantarum CRL 2130 mediated fermented soymilk (RLFSM) was studied using trinitrobenzene sulfonic induced colitis mouse model. RLFSM treatment significantly reduced the weight loss, intestinal damages, microbial translocation, and cytokine levels in the treated mice compared to controls. The study suggested that RLFSM might have anti-colitis property (Levit et al., 2017).

L. rhamnosus mediated fermented soymilk supplementation increased the isoflavone metabolites (O-desmethylangolensin and equol.) and short-chain fatty acids (propionic acid) content in mice. The fecal enzyme activity and amendment in gut microbiota were improved upon fermented soymilk administration. The study proved that the consumption of $L$. rhamnosus mediated fermented soymilk could improve the antibiotic-mediated gut microbial damages (Dai et al., 2019). 


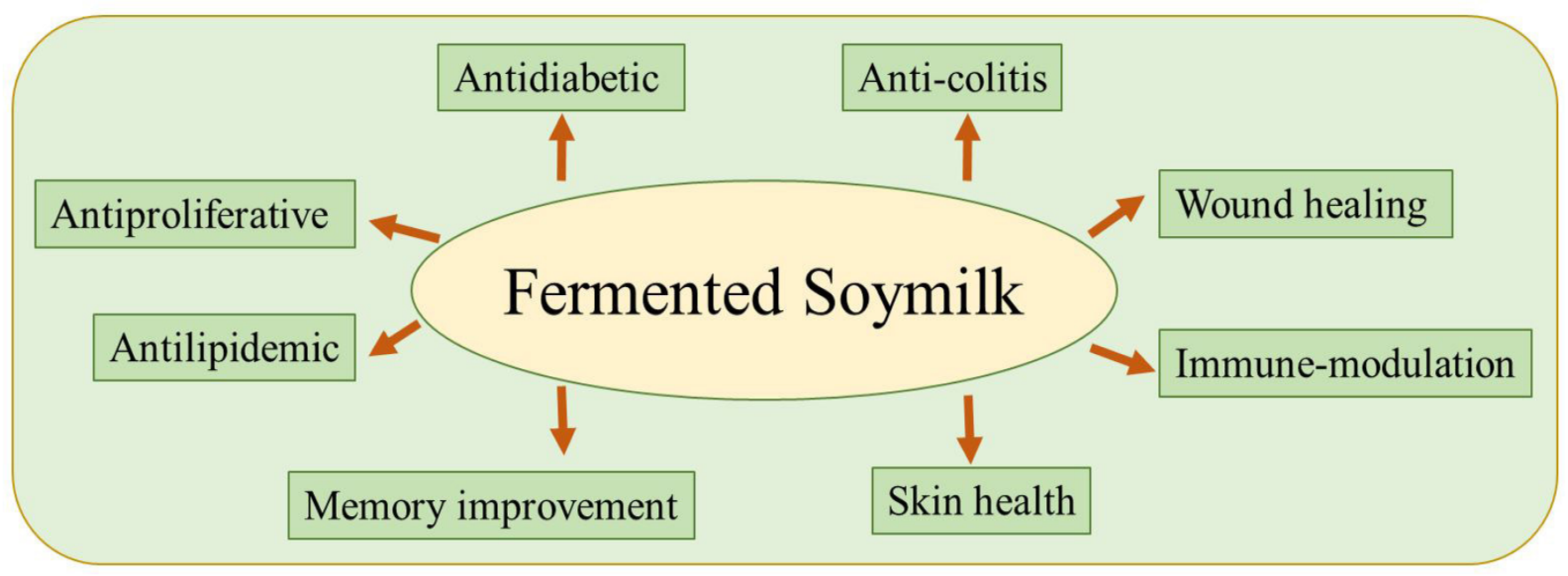

Figure 1. The major functional properties of fermented soymilk.

\section{Conclusion}

As per the literature survey, fermented soymilk has been reported for its antioxidant, antiproliferative, antidiabetic, hypolipidemic, immune-modulatory, memory improvement, and wound healing activities, etc. (Figure 1). However, most of the reports are based on in vivo and in vitro studies. A minimal number of studies have been carried out on human subjects. This research gap has to be filled to conclude that fermented soymilk products provide health benefits to human beings. Thus, further detailed studies are required in human subjects and there is a need to develop functionally superior fermented soymilk using potent starter cultures.

\section{Acknowledgements}

We wish to thank the Faculty of Pharmacy, Chiang Mai University, Chiang Mai, Thailand for the support. P. K. acknowledges the Chiang Mai University-Post-Doc grant for the necessary support.

\section{References}

Abe, S. K., Sawada, N., Ishihara, J., Takachi, R., Mori, N., Yamaji, T., Shimazu, T., Goto, A., Iwasaki, M., Inoue, M., \& Tsugane, S. (2020). Comparison between the impact of fermented and unfermented soy intake on the risk of liver cancer: the JPHC Study. European Journal of Nutrition. http://dx.doi.org/10.1007/s00394-020-02335-9. PMid:32719984.

Ahn, S. B., Wu, W. H., Lee, J. H., Jun, D. W., Kim, J., Kim, R., Lee, T. B., \& Jun, J. H. (2018). Fermented soymilk alleviates lipid accumulation by inhibition of SREBP-1 and activation of NRF-2 in the hepatocellular steatosis model. Journal of Microbiology and Biotechnology, 28(2), 236-245. http://dx.doi.org/10.4014/jmb.1707.07061. PMid:29169220.

Ahsan, S., Zahoor, T., Shehzad, A., \& Zia, M. A. (2019). Valuation of co-culture soymilk as a pragmatic approach on hyperglycemia and hypercholesterolemia in sprague-dawley rats. Journal of Animal and Plant Sciences, 29(3), 674-683.
Asif, M., \& Acharya, M. (2013). Phytochemicals and nutritional health benefits of soy plant. The C++ Report, 3(1), 64-69. http://dx.doi. org/10.4103/2231-0738.106998.

Babashahi, M., Mirlohi, M., Ghiasvand, R., \& Azadbakht, L. (2015). Comparison of soymilk and probiotic soymilk effects on serum high-density lipoprotein cholesterol and low-density lipoprotein cholesterol in diabetic Wistar rats. ARYA Atherosclerosis, 11(Suppl. 1), 6. PMid:26261455.

Babashahi, M., Mirlohi, M., Ghiasvand, R., Azadbakht, L., Mosharaf, L., \& Torki-Baghbadorani, S. (2020). Effects of probiotic soy milk fermented by Lactobacillus plantarum A7 (KC 355240) added with Cuminum Cyminum essential oil on fasting blood glucose levels, serum lipid profile and body weight in diabetic Wistar rats. International Journal of Preventive Medicine, 11(1), 8. http://dx.doi. org/10.4103/ijpvm.IJPVM_541_17. PMid:32089808.

Baú, T. R., Garcia, S., \& Ida, E. I. (2015). Changes in soymilk during fermentation with kefir culture: oligosaccharides hydrolysis and isoflavone aglycone production. International Journal of Food Sciences and Nutrition, 66(8), 845-850. http://dx.doi.org/10.3109/09637486 2015.1095861. PMid:26460145.

Chen, J., Wu, Y., Yang, C., Xu, X., \& Meng, Y. (2017). Antioxidant and hypolipidemic effects of soymilk fermented via Lactococcus acidophilus MF204. Food \& Function, 8(12), 4414-4420. http:// dx.doi.org/10.1039/C7FO00701A. PMid:29085943.

Chuang, Y. C., Cheng, M. C., Lee, C. C., Chiou, T. Y., \& Tsai, T. Y. (2019). Effect of ethanol extract from Lactobacillus plantarum TWK10fermented soymilk on wound healing in streptozotocin-induced diabetic rat. $A M B$ Express, 9(1), 163. http://dx.doi.org/10.1186/ s13568-019-0886-2. PMid:31605256.

Dai, S., Pan, M., El-Nezami, H. S., Wan, J. M. F., Wang, M. F., Habimana, O., Lee, J. C. Y., Louie, J. C. Y., \& Shah, N. P. (2019). Effects of lactic acid bacteria-fermented soymilk on isoflavone metabolites and short-chain fatty acids excretion and their modulating effects on gut microbiota. Journal of Food Science, 84(7), 1854-1863. http:// dx.doi.org/10.1111/1750-3841.14661. PMid:31206699.

Fakri, E. F. M., Lim, S. M., Musa, N. H., Hasan, M. H., Adam, A., \& Ramasamy, K. (2016). Lactobacillus fermentum LAB 9-Fermented soymilk with enriched isoflavones and antioxidants improved memory in vivo. Sains Malaysiana, 45(9), 1289-1297. 
Hsieh, M. L., \& Chou, C. C. (2006). Mutagenicity and antimutagenic effect of soymilk fermented with lactic acid bacteria and bifidobacteria. International Journal of Food Microbiology, 111(1), 43-47. http:// dx.doi.org/10.1016/j.ijfoodmicro.2006.04.034. PMid:16875752.

Hsieh, M. L., Fang, S. W., Yu, R. C., \& Chou, C. C. (2007). Possible mechanisms of anti-mutagenicity in fermented soymilk prepared with a coculture of Streptococcus infantis and Bifidobacterium infantis. Journal of Food Protection, 70(4), 1025-1028. http://dx.doi. org/10.4315/0362-028X-70.4.1025. PMid:17477279.

Jayachandran, M., \& Xu, B. (2019). An insight into the health benefits of fermented soy products. Food Chemistry, 271, 362-371. http:// dx.doi.org/10.1016/j.foodchem.2018.07.158. PMid:30236688.

Ju, H. E., \& Han, J. S. (2010). Hypoglycemic effect of fermented soymilk added with Bokbunja (Rubus coreanus Miquel) in diabetic mice. Food Science and Biotechnology, 19(4), 1041-1046. http://dx.doi. org/10.1007/s10068-010-0146-3.

Kano, M., Haga, K., Miyazaki, K., \& Ishikawa, F. (2018). Daily consumption of fermented soymilk helps to improve facial wrinkles in healthy postmenopausal women in a randomized, parallel-group, open-label trial. Functional Foods in Health and Disease, 8(2), 107-121. http:// dx.doi.org/10.31989/ffhd.v8i2.412.

Kano, M., Kubota, N., Masuoka, N., Hori, T., Miyazaki, K., \& Ishikawa, F. (2016). Oral Administration of fermented soymilk products protects the skin of hairless mice against ultraviolet damage. Nutrients, 8(8), 514. http://dx.doi.org/10.3390/nu8080514. PMid:27556484.

Kim, N. H., Moon, P. D., Kim, S. J., Choi, I. Y., An, H. J., Myung, N. Y., Jeong, H. J., Um, J. Y., Hong, S. H., \& Kim, H. M. (2008). Lipid profile lowering effect of Soypro ${ }^{\text {tw }}$ fermented with lactic acid bacteria isolated from Kimchi in high-fat diet-induced obese rats. BioFactors (Oxford, England), 33(1), 49-60. http://dx.doi.org/10.1002/ biof.5520330105. PMid:19276536.

Kitawaki, R., Nishimura, Y., Takagi, N., Iwasaki, M., Tsuzuki, K., \& Fukuda, M. (2009). Effects of lactobacillus fermented soymilk and soy yogurt on hepatic lipid accumulation in rats fed a cholesterol-free diet. Bioscience, Biotechnology, and Biochemistry, 73(7), 1484-1488. http://dx.doi.org/10.1271/bbb.80753. PMid:19584552.

Kobayashi, M., Harada, T., Takagi, N., Tsuzuki, K., Sugawara, M., \& Fukuda, M. (2012a). Effects of lactic acid-fermented soymilk on lipid metabolism-related gene expression in rat liver. Bioscience, Biotechnology, and Biochemistry, 76(1), 19-24. http://dx.doi. org/10.1271/bbb.100354. PMid:22232236.

Kobayashi, M., Hirahata, R., Egusa, S., \& Fukuda, M. (2012b). Hypocholesterolemic effects of lactic acid-fermented soymilk on rats fed a high cholesterol diet. Nutrients, 4(9), 1304-1316. http:// dx.doi.org/10.3390/nu4091304. PMid:23112918.

Kwon, Y., Apostolidis, E., \& Shetty, K. (2006). Anti-diabetes functionality of kefir culture-mediated fermented soymilk supplemented with Rhodiola extracts. Food Biotechnology, 20(1), 13-29. http://dx.doi. org/10.1080/08905430500522055.

Le, B., Ngoc, A. P. T., \& Yang, S. H. (2020). Synbiotic fermented soymilk with Weissella cibaria FB069 and xylooligosaccharides prevents proliferation in human colon cancer cells. Journal of Applied Microbiology, 128(5), 1486-1496. http://dx.doi.org/10.1111/ jam.14551. PMid:31834648.

Levit, R., de Giori, G. S., de Moreno de LeBlanc, A., \& LeBlanc, J. G. (2017). Evaluation of the effect of soymilk fermented by a riboflavinproducing Lactobacillus plantarum strain in a murine model of colitis. Beneficial Microbes, 8(1), 65-72. http://dx.doi.org/10.3920/ BM2016.0063. PMid:27873546.

Liao, C.L., Huang, H.Y., Sheen, L.Y., \& Chou, C.C. (2010). Antiinflammatory activity of soymilk and fermented soymilk prepared with lactic acid bacterium and Bifidobacterium. Journal of Food and Drug Analysis, 18(3), 202-210.

Liu, T. H., Chiou, J., \& Tsai, T. Y. (2016). Effects of Lactobacillus plantarum TWK10-fermented soymilk on deoxycorticosterone acetate-saltinduced hypertension and associated dementia in rats. Nutrients, 8(5), 260. http://dx.doi.org/10.3390/nu8050260. PMid:27144579.

Liu, T. H., Lin, W. J., Cheng, M. C., \& Tsai, T. Y. (2020). Lactobacillus plantarum TWK10-fermented soymilk improves cognitive function in type 2 diabetic rats. Journal of the Science of Food and Agriculture, 100(14), 5152-5161. http://dx.doi.org/10.1002/jsfa.10564. PMid:32529660.

Marazza, J. A., LeBlanc, J. G., de Giori, G. S., \& Garro, M. S. (2013). Soymilk fermented with Lactobacillus rhamnosus CRL981 ameliorates hyperglycemia, lipid profiles and increases antioxidant enzyme activities in diabetic mice. Journal of Functional Foods, 5(4), 18481853. http://dx.doi.org/10.1016/j.jff.2013.09.005.

Marazza, J. A., Nazareno, M. A., de Giori, G. S., \& Garro, M. S. (2012). Enhancement of the antioxidant capacity of soymilk by fermentation with Lactobacillus rhamnosus. Journal of Functional Foods, 4(3), 594-601. http://dx.doi.org/10.1016/j.jff.2012.03.005.

Messina, M. (2016). Soy and health update: Evaluation of the clinical and epidemiologic literature. Nutrients, 8(12), 754. http://dx.doi. org/10.3390/nu8120754. PMid:27886135.

Miyake, Y., Tanaka, K., Okubo, H., Sasaki, S., Furukawa, S., \& Arakawa, M. (2018). Soy isoflavone intake and prevalence of depressive symptoms during pregnancy in Japan: baseline data from the Kyushu Okinawa Maternal and Child Health Study. European Journal of Nutrition, 57(2), 441-450. http://dx.doi.org/10.1007/s00394-0161327-5. PMid:27744546.

Mohammadi Sartang, M., Mazloomi, S. M., Tanideh, N., \& Rezaian Zadeh, A. (2015). The effects of probiotic soymilk fortified with omega- 3 on blood glucose, lipid profile, hematological and oxidative stress, and inflammatory parameters in streptozotocin nicotinamide-induced diabetic Rats. Journal of Diabetes Research, 696372, 696372. http:// dx.doi.org/10.1155/2015/696372. PMid:26347893.

Myagmardorj, B., Purev, M. E., \& Batdorj, B. (2018). Functional properties of fermented soymilk by Lactobacillus fermentum BM325. Mongolian Journal of Chemistry, 19(45), 32-37. http://dx.doi. org/10.5564/mjc.v19i45.1087.

Nagino, T., Kaga, C., Kano, M., Masuoka, N., Anbe, M., Moriyama, K., Maruyama, K., Nakamura, S., Shida, K., \& Miyazaki, K. (2018). Effects of fermented soymilk with Lactobacillus casei Shirota on skin condition and the gut microbiota: a randomised clinical pilot trial. Beneficial Microbes, 9(2), 209-218. http://dx.doi.org/10.3920/ BM2017.0091. PMid:29264969.

Pisani, P., Parkin, D. M., Bray, F., \& Ferlay, J. (1999). Estimates of the worldwide mortality from 25 cancers in 1990. International Journal of Cancer, 83(1), 18-29. http://dx.doi.org/10.1002/(SICI)10970215(19990924)83:1<18::AID-IJC5>3.0.CO;2-M. PMid:10449602.

Qian, F., Zhang, J., Hou, K., Zhang, Y., Wang, Z., Luo, P., \& Tuo, Y. (2020). In vitro study of the antioxidative and antiproliferative capabilities of Lactobacillus casei 16-fermented soymilk. Food Science \& Nutrition, 8(1), 48-57. http://dx.doi.org/10.1002/fsn3.1214. PMid:31993131.

Seo, K. C., Kim, M. J., Hong, S. H., Cha, S. Y., Noh, J. S., Kim, M. J., \& Song, Y. O. (2010). The hypocholesterolemic effects of soymilk fermented with bacillus subtilis compared to soymilk with cheonggukjang powder in apolipoprotein-e knockout mice. Journal of Food Science and Nutrition, 15(2), 83-87.

Seo, K. C., Kim, M. J., Kwon, M. J., Kim, H. J., Noh, J. S., \& Song, Y. O. (2009). Antioxidative activities of soymilk fermented with Bacillus subtilis. Food Science and Biotechnology, 18(5), 1298-1300. 
Setiawan, I., Adriani, L., Zakiaturrahmi, Goenawan, H., Murniati Tarawan, V., \& Lesmana, R. (2020). Effect of different cow milk and soymilk (Soy yogurt) formulation on blood glucose level and glut4 gene expression in rats soleus muscle. Pakistan Journal of Biological Sciences, 23(12), 1607-1613. http://dx.doi.org/10.3923/ pjbs.2020.1607.1613. PMid:33274893.

Sirilun, S., Chaiyasut, C., Kesika, P., Peerajan, S., \& Sivamaruthi, B. S. (2017). Screening of lactic acid bacteria with immune modulating property, and the production of lactic acid bacteria mediated fermented soymilk. National Journal of Physiology, Pharmacy and Pharmacology, 7(12), 1397-1405. http://dx.doi.org/10.5455/ njppp.2017.7.0933926092017.

Tsai, J. S., Lin, Y. S., Pan, B. S., \& Chen, T. J. (2006). Antihypertensive peptides and $\gamma$-aminobutyric acid from prozyme 6 facilitated lactic acid bacteria fermentation of soymilk. Process Biochemistry, 41(6), 1282-1288. http://dx.doi.org/10.1016/j.procbio.2005.12.026.

Tsai, T., Chu, L., Lee, C., \& Pan, T. (2009). Atherosclerosis-preventing activity of lactic acid bacteria-fermented milk-soymilk supplemented with Momordica charantia. Journal of Agricultural and Food Chemistry, 57(5), 2065-2071. http://dx.doi.org/10.1021/jf802936c. PMid:19216552.

Usha Rani, V., \& Pradeep, B.V. (2015). Antioxidant properties of soy milk fermented with Lactobacillus paracasei KUMBB005. International Journal of Pharmaceutical Sciences, 30(1), 08.

Wagar, L. E., Champagne, C. P., Buckley, N. D., Raymond, Y., \& GreenJohnson, J. M. (2009). Immunomodulatory properties of fermented soy and dairy milks prepared with lactic acid bacteria. Journal of Food Science, 74(8), M423-M430. http://dx.doi.org/10.1111/j.17503841.2009.01308.x. PMid:19799669.

Wang, T. (2008). Minor constituents and phytochemicals of soybeans. In L. A. Johnson, P. J. White \& R. Galloway (Eds.), Soybeans: chemistry, production, processing, and utilization (pp. 297-330). Urbana: AOCS Press. http://dx.doi.org/10.1016/B978-1-893997-64-6.50013-5.

Wang, Y. C., Yu, R. C., \& Chou, C. C. (2006). Antioxidative activities of soymilk fermented with lactic acid bacteria and bifidobacteria. Food Microbiology, 23(2), 128-135. http://dx.doi.org/10.1016/j. fm.2005.01.020. PMid:16942996.

Wang, Z., Bao, Y., Zhang, Y., Zhang, J., Yao, G., Wang, S., \& Zhang, H. (2013). Effect of soymilk fermented with Lactobacillus plantarum P-8 on lipid metabolism and fecal microbiota in experimental hyperlipidemic rats. Food Biophysics, 8(1), 43-49. http://dx.doi. org/10.1007/s11483-012-9282-z.

Wu, S. J., Fang, J. Y., Ng, C. C., Wang, C. Y., \& Shyu, Y. T. (2013). Antiinflammatory activity of Lactobacillus-fermented adlay-soymilk in LPS-induced macrophages through suppression of NF- $\kappa \mathrm{B}$ pathways. Food Research International, 52(1), 262-268. http://dx.doi. org/10.1016/j.foodres.2013.02.053.

Xu, Y., Chen, X., Lu, M., Yang, Z., Huang, Y., Liu, D., Xiao, L., Sun, Y., Gu, W., Xu, D., \& Gu, R. (2012). In vitro and in vivo studies on the antioxidant effects of soymilk fermented with Streptococcus thermophilus grx02. Food Biotechnology, 26(4), 339-350. http:// dx.doi.org/10.1080/08905436.2012.724039.

Yamamoto, N., Shoji, M., Hoshigami, H., Watanabe, K., Watanabe, K., Takatsuzu, T., Yasuda, S., Igoshi, K., \& Kinoshita, H. (2019). Antioxidant capacity of soymilk yogurt and exopolysaccharides produced by lactic acid bacteria. Bioscience of Microbiota, Food and Health, 38(3), 97-104. http://dx.doi.org/10.12938/bmfh.18-017. PMid:31384521.

Yeom, S. J., Kim, B. N., Kim, Y. S., \& Oh, D. K. (2012). Hydrolysis of isoflavone glycosides by a thermostable $\beta$-glucosidase from Pyrococcus furiosus. Journal of Agricultural and Food Chemistry, 60(6), 15351541. http://dx.doi.org/10.1021/jf204432g. PMid:22251001.

Yi, N., Hwang, J. Y., \& Han, J. S. (2009). Hypoglycemic effect of fermented soymilk extract in stz-induced diabetic mice. Journal of Food Science and Nutrition, 14(1), 8-13.

Zhai, Q., Xiao, Y., Tian, F., Wang, G., Zhao, J., Liu, X., Chen, Y. Q., Zhang, H., \& Chen, W. (2015). Protective effects of lactic acid bacteriafermented soymilk against chronic cadmium toxicity in mice. RSC Advances, 5(6), 4648-4658. http://dx.doi.org/10.1039/C4RA12865F.

Zhang, X., Shu, X. O., Gao, Y. T., Yang, G., Li, Q., Li, H., Jin, F., \& Zheng, W. (2003). Soy food consumption is associated with lower risk of coronary heart disease in Chinese women. The Journal of Nutrition, 133(9), 2874-2878. http://dx.doi.org/10.1093/jn/133.9.2874. PMid:12949380.

Zhang, X. L., Wu, Y. F., Wang, Y. S., Wang, X. Z., Piao, C. H., Liu, J. M., Liu, Y. L., \& Wang, Y. H. (2017). The protective effects of probioticfermented soymilk on high-fat diet-induced hyperlipidemia and liver injury. Journal of Functional Foods, 30, 220-227. http://dx.doi. org/10.1016/j.jff.2017.01.002. 